\title{
A New Hardware Correlator in Korea: Performance Evaluation USing KVN OBSERVATions
}

\author{
Sang-Sung Lee ${ }^{1,2}$, Chung Sik OH ${ }^{1}$, Duk-Gyoo Roh ${ }^{1}$, Se-Jin OH ${ }^{1}$, Jongsoo Kim ${ }^{1,2}$, Jae-Hwan Yeom ${ }^{1}$, \\ Hyo Ryoung Kim ${ }^{1}$, Dong-Gyu Jung ${ }^{1}$, Do-Young Byun ${ }^{1}$, Taehyun Jung ${ }^{1,2}$, Noriyuki Kawaguchi ${ }^{3}$, \\ KatsunORi M. Shibata ${ }^{4}$, AND KiYOAKI WAJima ${ }^{1}$ \\ ${ }^{1}$ Korea Astronomy and Space Science Institute, 776 Daedeokdae-ro, Yuseong-gu, Daejeon 305-348, Korea \\ sslee@kasi.re.kr \\ ${ }^{2}$ Korea University of Science and Technology, 176 Gajeong-dong, Yuseong-gu, Daejeon 305-350, Korea \\ ${ }^{3}$ Shanghai Observatory, Chinese Academy of Sciences, 80 Nandan Road, 200030 Shanghai, P.R. China \\ ${ }^{4}$ Mizusawa VLBI Observatory, National Astronomical Observatory of Japan, 2-21-1 Osawa, Mitaka, Tokyo 181-8588, Japan
}

Received February 9, 2015; accepted March 16, 2015

\begin{abstract}
We report results of the performance evaluation of a new hardware correlator in Korea, the Daejeon correlator, developed by the Korea Astronomy and Space Science Institute (KASI) and the National Astronomical Observatory of Japan (NAOJ). We conduct Very Long Baseline Interferometry (VLBI) observations at $22 \mathrm{GHz}$ with the Korean VLBI Network (KVN) in Korea and the VLBI Exploration of Radio Astrometry (VERA) in Japan, and correlated the aquired data with the Daejeon correlator. For evaluating the performance of the new hardware correlator, we compare the correlation outputs from the Daejeon correlator for KVN observations with those from a software correlator, the Distributed FX (DiFX). We investigate the correlated flux densities and brightness distributions of extragalactic compact radio sources. The comparison of the two correlator outputs shows that they are consistent with each other within $<8 \%$, which is comparable with the amplitude calibration uncertainties of KVN observations at $22 \mathrm{GHz}$. We also find that the $8 \%$ difference in flux density is caused mainly by (a) the difference in the way of fringe phase tracking between the DiFX software correlator and the Daejeon hardware correlator, and (b) an unusual pattern (a double-layer pattern) of the amplitude correlation output from the Daejeon correlator. The visibility amplitude loss by the double-layer pattern is as small as $3 \%$. We conclude that the new hardware correlator produces reasonable correlation outputs for continuum observations, which are consistent with the outputs from the DiFX software correlator.
\end{abstract}

Key words: Techniques: interferometric — Instrumentation: interferometers — Radio continuum: galaxies - Masers

\section{INTRODUCTION}

Very Long Baseline Interferometry (VLBI) is an astronomical observing technique developed in the 1960s for measuring the accurate position of compact radio sources and obtaining their sky brightness distribution at high angular resolution by detecting fringes of noise signals arriving at two, or more, radio telescopes from the celestial compact radio sources (Ryle \& Hewish 1960; Clark et al. 1967; Moran et al. 1967). The noise signals arriving at each radio telescope are filtered, down-converted, and digitally sampled with being accurately time-tagged by a frequency standard located at each observatory. The sampled signals are recorded using magnetic tape systems such as the Mark I system (Bare et al. 1967), the Mark II system (Clark 1973), the Mark III system (Rogers et al. 1983), the Mark IV system (Whitney 1993), and the S2 system (Wietfeldt et al. 1996), or to hard disk systems such as the Mark 5 system (Whitney 2002) and the K5 system (Kondo et al. 2003). The recording media are shipped to a correlator center and played back. At that moment, one of the

CORRESPONDING AUThOR: S.-S. Lee main roles of the correlator is to detect a fringe between the two signals from radio telescopes after correcting for geometric and instrumental delays of the signals. Pre-estimates of the delays, apriori data are applied to the correlation of the signals from the two radio telescopes (or from a baseline). The correlation output in this stage is known as the visibility. The correlation output streams are formatted in Flexible Image Transport System (FITS) interferometry Data Interchange Convention (Greisen 2009). Through a post-correlation process, the visibilities are used for measuring the accurate positions of celestial radio sources and recovering their sky brightness distributions. The post-correlation process is performed generally with astronomical processing programs: for example, the Astronomical Image Processing System (AIPS).

A number of VLBI correlators have been developed to detect the fringes from VLBI observations. The VLBI correlators include hardware and software correlators (Bare et al. 1967; Moran et al. 1967; Napier et al. 1994; Wilson et al. 1996; Casse 1999; Carlson 1999; Horiuchi et al. 2000; Deller et al. 2007, 2011). Technical progress in parallel computing and high-speed net- 
works enables the construction of software correlators at low price and with short development period. Especially software correlator such as the DiFX (Deller et al. 2007, 2011) became popular because of its easy installation and extensive support from its user groups. The price per baseline and price per unit data bandwidth are almost the same in software correlators, while the prices decrease significantly with the number of baseline and data rate in hardware correlators. Therefore, hardware correlators become attractive when the target VLBI array consists of a large number of antennas with a wide bandwith system. In addition, the electric power consumption of hardware correlators is much less than that of software correlators, significantly reducing the total operation budget. These are the main reasons why connected arrays, which usually have more elements and wider bandwidth than VLBI arrays, still prefer hardware correlators.

A new hardware correlator (the Daejeon correlator, Figure 1) was developed in 2006 - 2009 by the Korea Astronomy and Space Science Institute (KASI) and the National Astronomical Observatory of Japan (NAOJ). The Daejeon correlator will be the main correlator for the East Asian VLBI Network (EAVN) consisting of the Korean VLBI Network (KVN) in Korea (Lee et al. 2011, 2014), the Japanese VLBI Network (JVN) including the VLBI Exploration of Radio Astrometry (VERA) in Japan, and the Chinese VLBI Network (CVN). This is one of the main motivations for developing the new hardware correlator rather than adopting a software correlator like DiFX (Deller et al. 2007, 2011). The capability of efficient correlation for large number of stations (e.g., >20 for EAVN stations at $22 \mathrm{GHz}$ ) is one of the advantages of the hardware correlator. In 2010 we started commissioning operations of the correlator, and began to evaluate its performance using test observations with the combined network of KVN and VERA (Figure 2).

The Daejeon hardware correlator is located in the Korea-Japan Correlation Center (KJCC), Korea. The Daejeon correlator consists of several VLBI data playback systems, a Raw VLBI Data Buffer (RVDB), a VLBI Correlation Subsystem (VCS), and a data archive system. The Daejeon correlator was aimed to correlate data obtained from various VLBI networks in East Asia: the KVN, the KVN and VERA combined network, and the East Asia VLBI Network. As these use different recording systems, the Daejeon correlator has several VLBI data playback systems: Mark 5B, VERA2000, OCTADISK, etc. They have different interface for data transmission. The difference in the formats of the playback system led to the introducion of the RVDB system, a big data server with several interfaces. Data from the RVDB are transferred to the VCS, the main part of the Daejeon correlator, and correlated with proper control parameters provided by the correlator control and operation computers. The VCS is able to process a maximum of 16 stations with a maximum recording rate of $8192 \mathrm{Mbps}$, and 8192 output channels for VLBI observation data. The correlation results

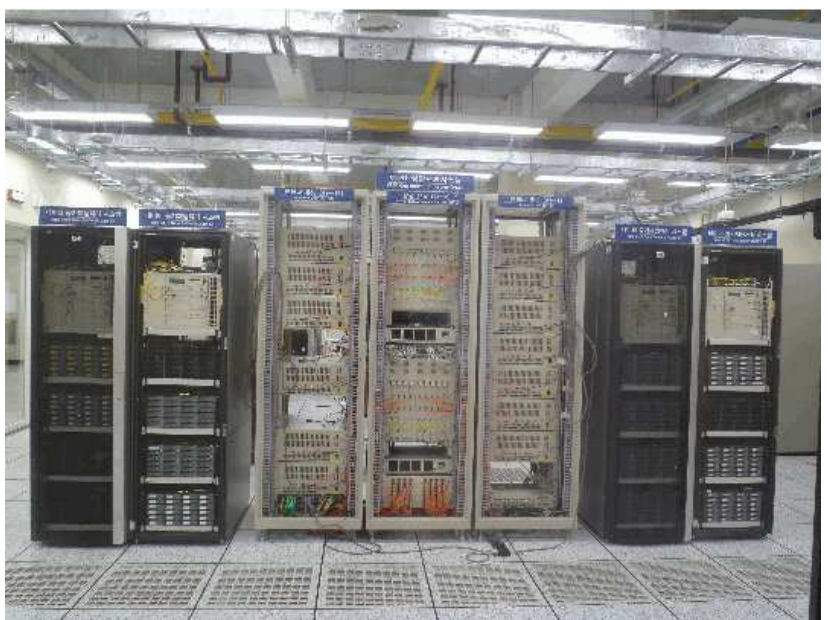

Figure 1. Daejeon correlator (KASI, Daejeon). Adopted from Lee et al. (2014)

from the VCS are then transferred to the data archive system. Detailed description of the correlator will be presented elsewhere (Oh et al. 2015, in preparation), and its current status can be found in the Internet. ${ }^{\mathbf{1}}$

Performance of the new correlator can be evaluated via investigating the outputs from the whole cycle of the VLBI observations: data acquisition, correlation, and post-correlation process. Careful comparison of the output from the new correlator with that from an existing, reliable correlator (e.g., DiFX as described in Deller et al. 2007, 2011) should be conducted.

We conducted test observations of compact radio sources using KVN and VERA, correlated the observed data with the Daejeon correlator and the DiFX software correlator, and performed careful comparison of the outputs. An extensive evaluation using the Daejeon correlator, the DiFX correlator, and Mitaka FX correlator in NAOJ (Shibata et al. 1998) for the KVN and VERA observations has been conducted. Full results of the extensive comparison will be reported elsewhere (Oh et al. 2015, in preparation). In this paper, we report the results of the comparison between the Daejeon correlator and the DiFX correlator using the observations only with the KVN. In Section 2, we describe the observations, correlation, and the post-correlation process. The results of this comparison are reported in Section 3, and we discuss an unusual pattern of the correlation output in Section 4. We make conclusions in Section 5.

\section{OBSERVATIONS AND DATA REDUCTION}

\subsection{Observations Using KVN and VERA}

We observed compact radio sources, 3C 454.3, 3C 345, NRAO 512 and Sagittarius A* (Sgr A*) at $22 \mathrm{GHz}$, on 2011 January 28 UT 19:00 and January 29 UT 04:00, using KVN and VERA (7 stations): KVN Yonsei (KY), KVN Ulsan (KU), KVN Tamna (KT), Mizu-

\footnotetext{
${ }^{1}$ http://kjcc.kasi.re.kr
} 


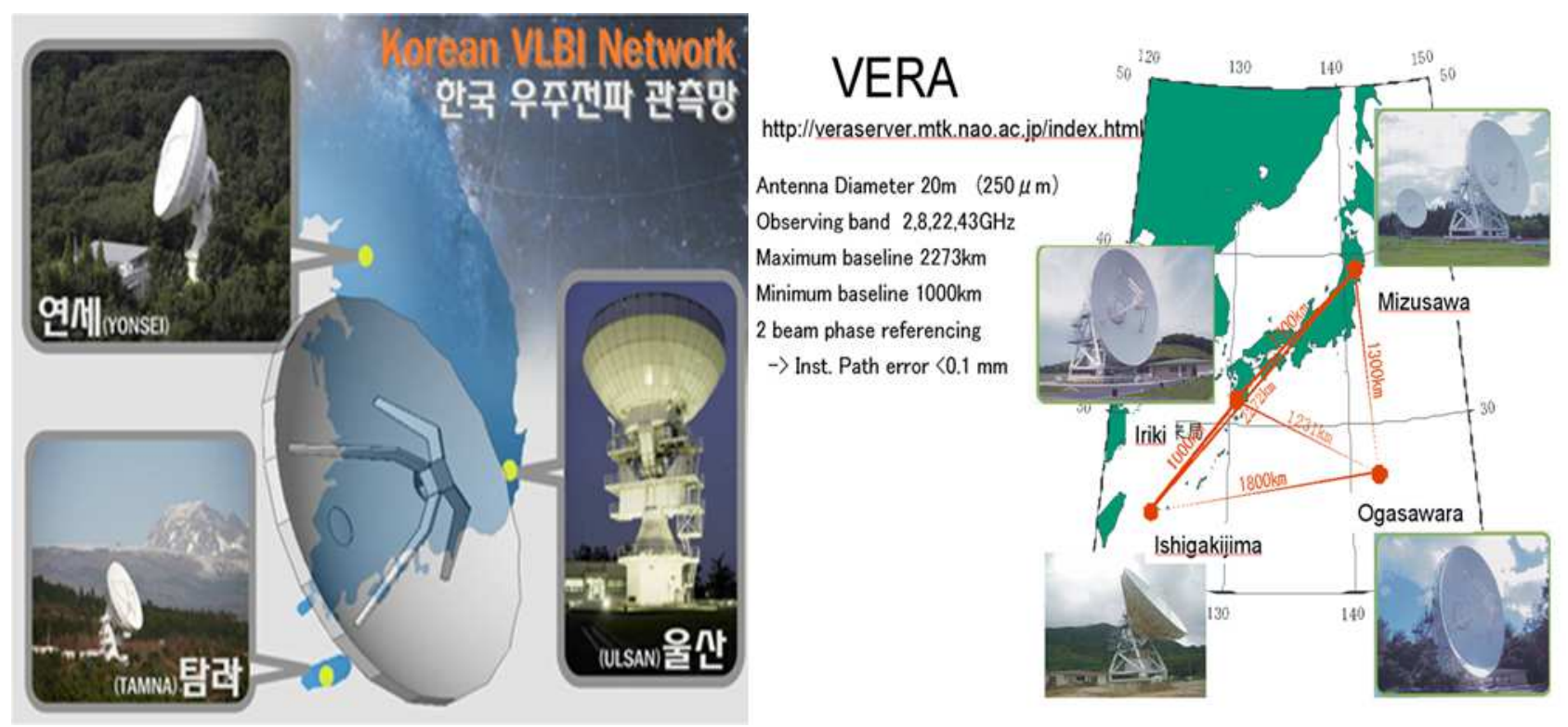

Figure 2. Korean VLBI Network (KVN) operated by KASI, Korea (left) and VLBI Exploration of Radio Astrometry (VERA) operated by NAOJ, Japan (right).

sawa (MIZ), Ishigakigima (ISH), Ogasawara (OGA), and Iriki (IRK). In order to evaluate the various correlation performances of the Daejeon correlator, the several observing modes and scan lengths were considered. For this comparison, we used longer scans (10-20 $\min$ ) for all sources. The observing frequency is 22.00 $22.45 \mathrm{GHz}$ with a selected bandwidth of $256 \mathrm{MHz}$ and in left circular polarization (LCP). The received signals within the frequency bandwidth are 2-bit quantized by $\mathrm{AD}$ converters located in the telescope cabin and transferred to the observing building via optical fibre. The transferred digital signals are divided into 16 sub-bands (IFs) by a digital filter bank, and recorded in magnetic disks or tapes by two data acquisition systems: the Mark 5B system (disk-based) in KVN and the VERA2000 system (tape-based) in VERA. The recording rate is $1024 \mathrm{Mbps}$.

\subsection{Correlation Using Daejeon Correlator and DiFX}

The recording media were sent to the KJCC. The shipped media were played back using Mark 5B for KVN data and VERA2000 for VERA data. The data from the playback systems were transferred to the RVDB to be correlated in the VCS. Finally, the data were correlated using a $1.6384 \mathrm{~s}$ correlator integration time and 128 spectral channels across each $16 \mathrm{MHz}$ band. The correlation was performed in 2013 July. The data recorded at KVN stations were also correlated with the DiFX software correlator. Each scan recorded in the Mark 5B disk packs was extracted by using the utility FUSE, $^{2}$ and was transferred to the file server through the 10 Gigabit network. Scans for strong sources were first used for searching the clock offset and rate of each station, and then all scans were correlated. The correlator integration time was $2.048 \mathrm{~s}$

\footnotetext{
$\mathbf{2}_{\text {http://fusemk5a.sourceforge.net }}$
}

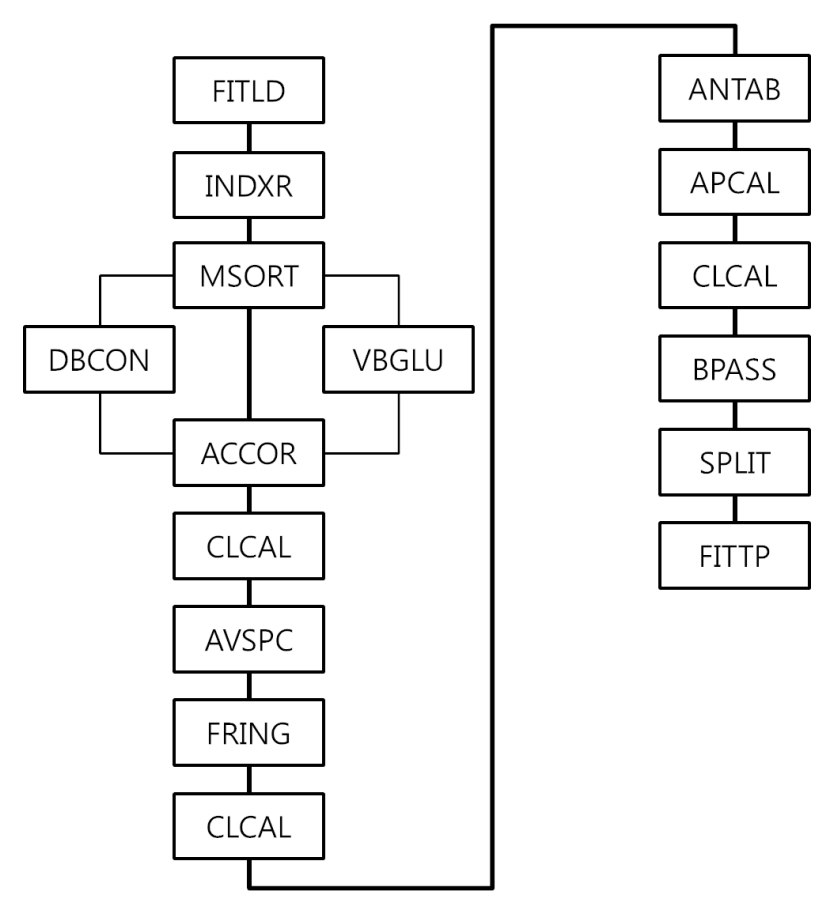

Figure 3. Schematic diagram of VLBI data reduction with AIPS

for DiFX and the number of spectral channels was 128 across each $16 \mathrm{MHz}$ band.

\subsection{Post-Correlation Process Using AIPS and DIFMAP}

We performed further processing using AIPS. We followed a standard processing procedure as described in Figure 3. The correlated output in FITS format was uploaded into an AIPS catalog by the FITLD task, and information of the FITS file were indexed with the INDXR task. In using FITLD, we did not apply 
digital correction (Digicor $=-1$ ), since it is not necessary for the two correlators. We sorted the indexed catalog in an order of time and baseline (TB) and if necessary, indexed them again. FITS files from the correlator that were divided into several files in time were combined using the DBCON task, while those divided in frequency were combined with the VBGLU task. At KVN and VERA, we sampled the received signals using the digital samplers. During the digital sampling, there could be some amplitude errors of crosscorrelation power spectrum. These errors can be corrected using the ACCOR task based on the amplitude of the auto-correlation power spectrum for each station. These corrections for the amplitudes are stored in a solution (SN) table, separate from the FITS file itself. In order to apply the correction in the SN table, we used the CLCAL task and generated a new calibration (CL) table. At this stage, one may average the FITS data in frequency using the AVSPC task. We did not average the FITS data for this work. The residuals of the fringe delay and delay rate after the correlation were searched using the FRING task. This is known as fringe-fitting. FRING provides us with antenna-based solutions of the delay and delay rate based on baseline-based solutions. The solution interval for the fringe-fitting should be pre-determined according to the coherence time of the observation at the observing frequency. For this observation at $22 \mathrm{GHz}$, we used $1 \mathrm{~min}$ as the solution interval of the fringe-fitting. Once we obtained the solutions of the delay and delay rate, we calibrated the phase using CLCAL. After the phase calibration, we performed the amplitude calibration in order to correct for the atmospheric opacity change and for the amplitude errors due to atmospheric fluctuations. We used system temperatures and antenna gain measured at each observatory for converting the correlation coefficient to sky brightness and correcting for the amplitude errors. We stored the calibration information in the TY and GC tables using the ANTAB task and produced the solution of the amplitude calibrations using the APCAL task. We did not correct for the effect of the bandpass filter on the spectrum shape in order to investigate the correlation effect on the spectrum shape. We divided the FITS data for each source with applying all calibration information and exported into $u v$-file using FITTP. For the purpose of the comparison of images, we used the central 115 channels of each IF, excluding the first 6 and last 7 channels.

After the phase and amplitude calibration, we made the contour maps of the target sources using the Caltech DIFMAP software (Shepherd et al. 1994). We averaged the $u v$-data (or visibility data) in a time interval of $30 \mathrm{~s}$, and flagged outliers in amplitude in order to increase the data processing speed and decrease random error of individual visibility data. We should note that the averaging time interval of $30 \mathrm{~s}$ is shorter than the coherence time of this observation. As a first step of mapping (or imaging) with DIFMAP, we fitted a point-source model to the visibility and self-calibrated the phase according to the model, in order to find the converged model to the visibility. As a second step, we used the CLEAN deconvolution algorithm and the amplitude- and phaseself-calibration alternatively. The CLEAN deconvolution is a technique to deconvolve the visibility data to find the true sky brightness distribution of the target source by establishing a group of delta-function models. The self-calibration is an algorithm to reduce the difference between the models and visibility phase/amplitude using closure phase and closure amplitude. We should note that the amplitude-self-calibration can not be performed for the observations with three or less stations. Therefore, for the analysis of these KVN data, we did not perform amplitude-self-calibration. We evaluated the quality of the final map, by investigating the residual noise in the image as described in Lobanov et al. (2006). We can quantitatively estimate the noise in the final CLEANed image based on the ratio of image noise rms to its mathematical expectation, $\xi_{r}$. Suppose that a residual image has an $\mathrm{rms} \sigma_{\mathrm{r}}$ and a maximum absolute flux density $\left|s_{\mathrm{r}}\right|$. For Gaussian noise with a zero mean, the expectation of $s_{\mathrm{r}}$ is

$$
\left|s_{\mathrm{r}, \exp }\right|=\sigma_{\mathrm{r}}\left[\sqrt{2} \ln \left(\frac{N_{\mathrm{pix}}}{\sqrt{2 \pi} \sigma_{\mathrm{r}}}\right)\right]^{1 / 2},
$$

where $N_{\text {pix }}$ is the total number of pixels in the image. The ratio $\xi_{r}$ is given by

$$
\xi_{\mathrm{r}}=s_{\mathrm{r}} / s_{\mathrm{r}, \exp }
$$

When the residual noise is similar to Gaussian noise, $\xi_{\mathrm{r}} \rightarrow 1$. If $\xi_{\mathrm{r}}>1$, not all the structure has been adequately cleaned or recovered; if $\xi_{\mathrm{r}}<1$, the image model obtained has an excessively large number of degrees of freedom. In order to compare quantitative information of the final images, we used circular Gaussiancomponent models to fit the self-calibrated data, yielding the following modelfit parameters: the total flux density $S_{\mathrm{t}}$, size $d$, radial distance $r$, and position angle $\theta$ of each component.

\section{Results}

\subsection{Visibility Comparison with DiFX}

After the calibration and data reduction, we compared the calibrated output of the Daejeon correlator with that of DiFX. For a careful comparison, we used the same parameters and data reduction procedures for the two cases, except for the correlator integration time (1.6384 s for Daejeon and $2.048 \mathrm{~s}$ for DiFX) and the delay models (a Mitaka delay model for Daejeon correlator and a CALC9 delay model for DiFX).

Figures $4-6$ show the visibility amplitudes on the sources, 3C 454.3, 3C 345, and NRAO 512 for IF 214 and three KVN baselines from both correlators as a function of time. The whole frequency channel (128) data have been vector-averaged. We fitted the visibility amplitudes for each baseline with a first-order polynomial $V(t)=A t+B$ using a linear least-squares method. The fitted models and averaged values are shown in Figures $4-6$. The averaged visibility amplitudes over 


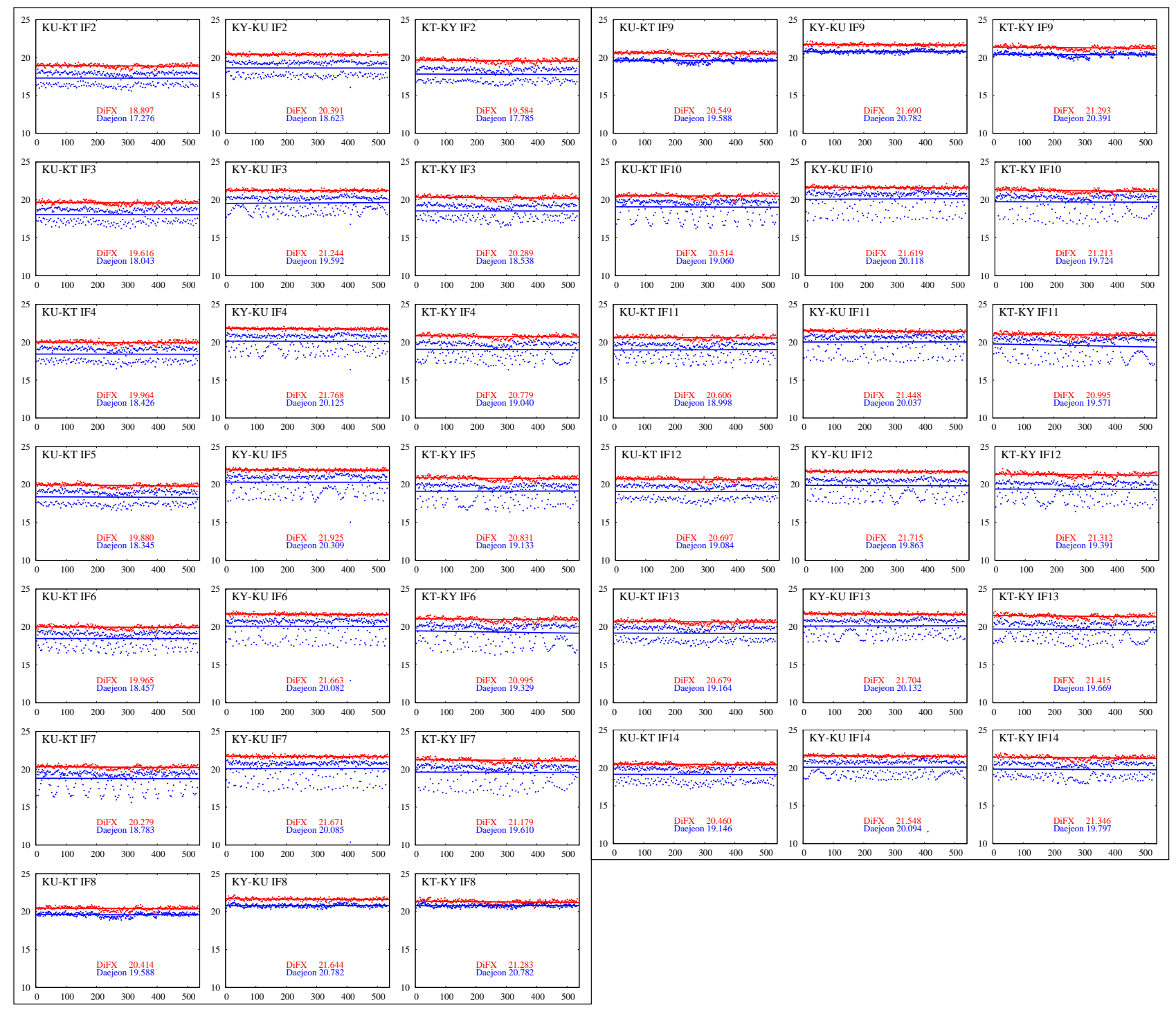

Figure 4. Comparison of the visibility amplitude as a function of time for the source 3C 454.3 as calculated by the DiFX (red dots) and the Daejeon correlators (blue dots) for IF 2-14 and three KVN baselines: KU-KT, KY-KU, and KT-KY during 9 min after 2011 January 29 04:20:00 UT. The visibility amplitude is in Jy and the time is in seconds from the start of the scan. The solid lines represent linear fits to the visibility amplitude for the DiFX (red line) and the Daejeon correlator (blue line). The numbers on each panel indicate the averaged amplitude for each correlator over the time period.

IF 2-14 within full frequency channels and central 115 channels for individual baselines and sources are summarized in Table 1. In Table 1, we summarized the mean visibility amplitudes of IF 2-14 for two cases of using full frequency channels in IF and of using only central 115 channels. The visibility amplitudes of the target sources are in the range of $0.5-22 \mathrm{Jy}$. The visibility amplitudes for the Daejeon correlator seem relatively lower than those for DiFX. We found that the averaged visibility amplitudes of individual baselines for the Daejeon correlator are lower by $\leq 8 \%$ than those of DiFX for all sources and all baselines.

The amplitude difference between two correlators can be attributed mainly to an unusual pattern of the visibility amplitude of the Daejeon correlator, so called double-layer pattern, as clearly shown in Figure 4. The double-layer pattern consists of two main patterns at higher and lower amplitude values. One pattern is similar to that of DiFX (normal pattern). The other has lower values in amplitude than the main pattern and sometimes is similar to a sinusoidal pattern. For the baseline KU-KT in IF 2 in Figure 4, one third of the visibility data are along the level of about $16 \mathrm{Jy}$, and the remaining data are at about $18 \mathrm{Jy}$. For the baseline KU-KT in IF 7 , one third of the visibility data show a sinusoidal pattern with its amplitude of about $2 \mathrm{Jy}$, that is about $15 \%$ of the flux density. The double-layer patterns appear different in IF (i.e., in frequency) and are present in all baselines and IF bands except for IF 8 and 9 . Among the IF $2-14$ for the baseline KU-KT, for IF $2-5$ and IF $12-15$ the double-layer patterns are shown as two parallel patterns, and for IF 6-7 and IF 


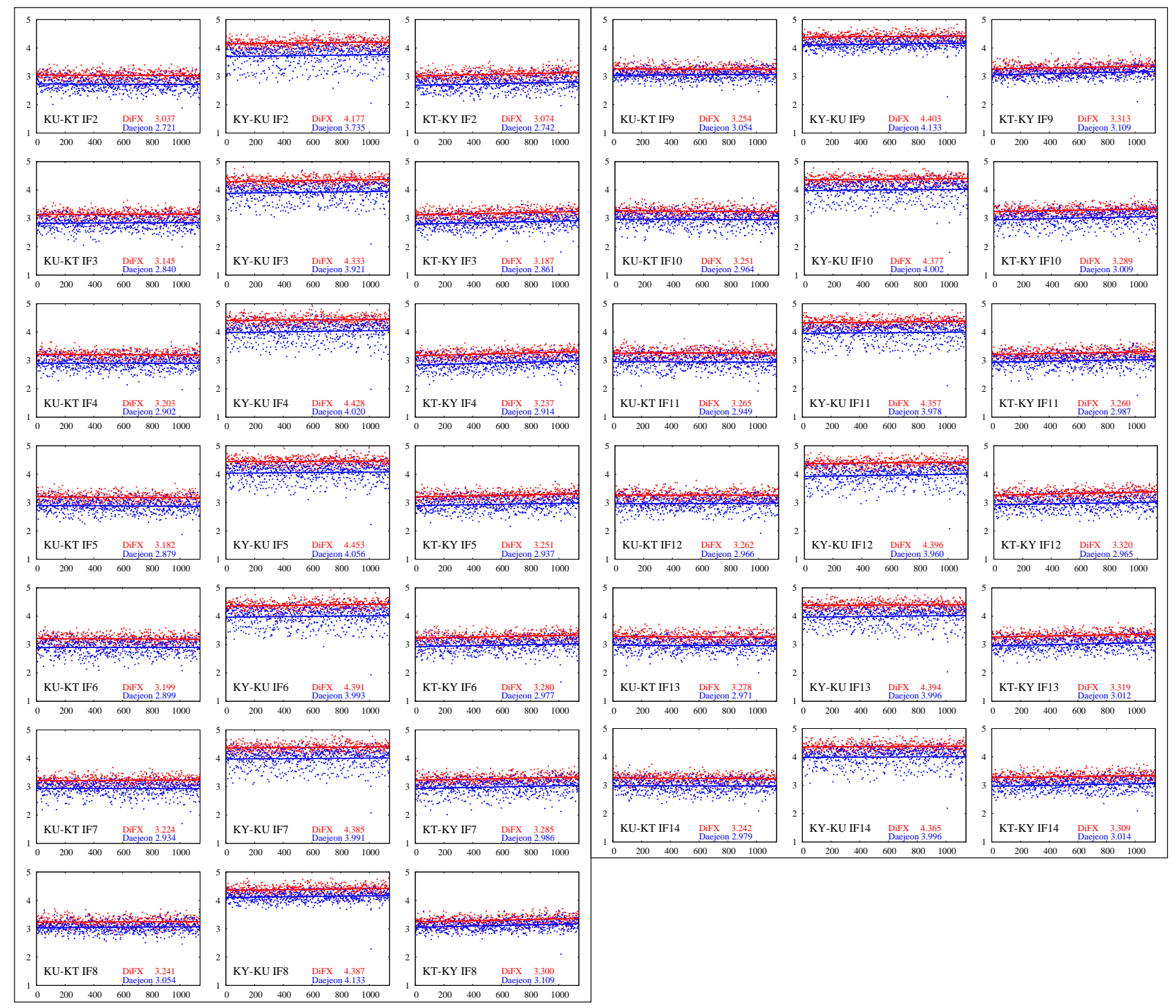

Figure 5. Same as Figure 4 but for the source 3C 345 and the time range during 19 min after 2011 January 28 19:30:00 UT.

10-11 the double-layer patterns appear like the sinusoidal pattern. The visibility amplitudes for IF 8 and 9 do not show such a double-layer pattern. It seems that the period of the sinusoidal pattern varies in time and frequency. The periods for IF 6 and 11 seems twice smaller than those for IF 7 and 10, respectively. We found also that there is a rough symmetric trend of the double-layer patterns between IF 2-8 and IF 9-15. This implies that the double-layer patterns may happen in a correlation stage related with frequency. The double-layer patterns are clearer for the observations of stronger sources like 3C 454.3 with a correlated flux density of $\sim 20 \mathrm{Jy}$ (Figure 4), whereas they are less clear for relatively weaker sources like 3C 345 and NRAO 512 with flux densities of 1-4 Jy (Figures 5 and 6 ). This is because the amplitude of the double-layer pattern is about $15 \%$ and it is similar to the amplitude calibration uncertainty of KVN observations at $22 \mathrm{GHz}$ (see Petrov et al. 2012; Lee et al. 2014). However, the 8\%-difference of visibility amplitudes between DiFX and the Daejeon correlator appears in all sources.

Figure 7 shows the visibility phases of the source 3C 454.3 for IF 2-14 and three KVN baselines from both correlators as a function of time. The whole frequency channel (128) data have been vector-averaged. We found that the visibility phases show small differences due to the different delay models used for the two correlators.

Figure 8 shows a comparison of the cross-correlated spectrum, i.e., the visibility amplitude and phases as a function of frequency in one $16 \mathrm{MHz}$ band, IF 2. The spectra have been vector-averaged over one minute time range. The phases of the cross-correlated spectrum are consistent with each other within $\leq 2$ degrees, whereas the amplitudes are different by about $10 \%$ on average. The amplitude difference varies in frequency. At the band edges, the difference gets larger, whereas at the band center, it becomes a bit smaller. This indicates that the spectrum shape of Daejeon correlator output is a bit different from that of DiFX. 


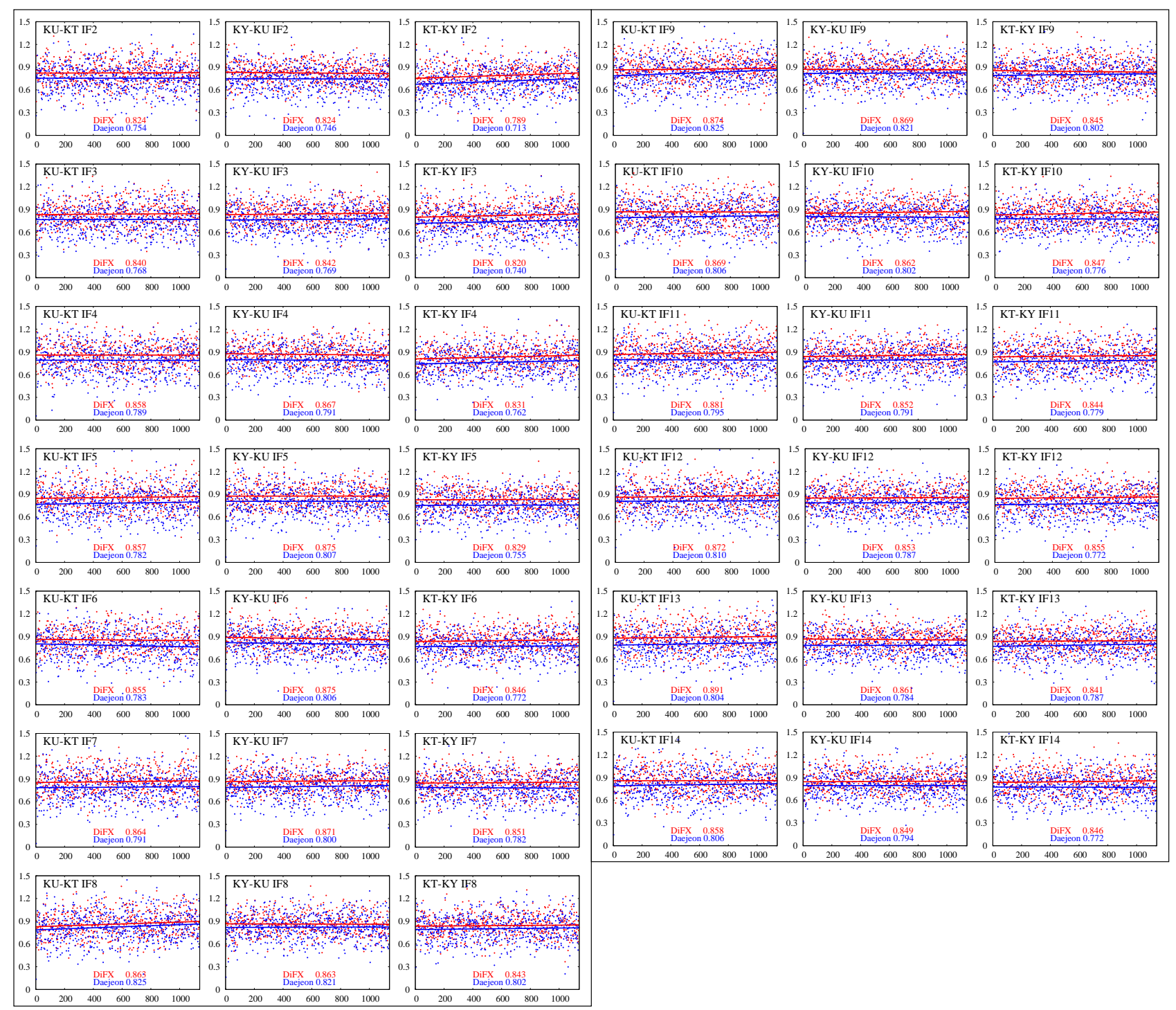

Figure 6. Same as Figure 4 but for the source NRAO 512 and the time range during 19 min after 2011 January 28 19:10:00 UT.

Table 1

Comparison of visibility amplitudes

\begin{tabular}{|c|c|c|c|c|c|c|c|}
\hline \multirow[b]{2}{*}{$\begin{array}{l}\text { Source } \\
(1)\end{array}$} & \multirow[b]{2}{*}{$\begin{array}{l}\text { Correlator } \\
(2)\end{array}$} & \multicolumn{2}{|c|}{$S_{\mathrm{KU}-\mathrm{KT}}$} & \multicolumn{2}{|c|}{$S_{\mathrm{KY}-\mathrm{KU}}$} & \multicolumn{2}{|c|}{$S_{\mathrm{KT}-\mathrm{KY}}$} \\
\hline & & $\begin{array}{c}\text { FULL BW } \\
\text { (3) }\end{array}$ & $\begin{array}{c}115 \mathrm{CH} \\
(4)\end{array}$ & $\begin{array}{c}\text { FULL BW } \\
(5)\end{array}$ & $\begin{array}{c}115 \mathrm{CH} \\
(6)\end{array}$ & $\begin{array}{l}\text { FULL BW } \\
(7)\end{array}$ & $\begin{array}{c}115 \mathrm{CH} \\
(8)\end{array}$ \\
\hline 3C 454.3 & $\begin{array}{l}\text { DiFX } \\
\text { Daejeon } \\
S_{\text {Daejeon }} / S_{\text {DiFX }}\end{array}$ & $\begin{array}{c}20.2 \pm 0.547 \\
18.8 \pm 1.04 \\
0.93(1.9)\end{array}$ & $\begin{array}{c}21.2 \pm 0.576 \\
19.8 \pm 1.09 \\
0.93(1.9)\end{array}$ & $\begin{array}{c}21.5 \pm 0.392 \\
20.0 \pm 1.07 \\
0.93(2.7)\end{array}$ & $\begin{array}{c}22.6 \pm 0.413 \\
21.1 \pm 1.13 \\
0.93(2.7)\end{array}$ & $\begin{array}{c}21.0 \pm 0.554 \\
19.4 \pm 1.17 \\
0.92(2.1)\end{array}$ & $\begin{array}{c}22.0 \pm 0.586 \\
20.4 \pm 1.23 \\
0.93(2.1)\end{array}$ \\
\hline 3C 345 & $\begin{array}{l}\text { DiFX } \\
\text { Daejeon } \\
S_{\text {Daejeon }} / S_{\text {DiFX }}\end{array}$ & $\begin{array}{c}3.21 \pm 0.169 \\
2.93 \pm 0.236 \\
0.91(1.3)\end{array}$ & $\begin{array}{c}3.37 \pm 0.181 \\
3.09 \pm 0.252 \\
0.92(1.4)\end{array}$ & $\begin{array}{c}4.37 \pm 0.158 \\
3.99 \pm 0.291 \\
0.91(1.84)\end{array}$ & $\begin{array}{c}4.59 \pm 0.170 \\
4.21 \pm 0.308 \\
0.92(1.8)\end{array}$ & $\begin{array}{c}3.26 \pm 0.167 \\
2.97 \pm 0.239 \\
0.91(1.4)\end{array}$ & $\begin{array}{c}3.43 \pm 0.179 \\
3.13 \pm 0.255 \\
0.91(1.4)\end{array}$ \\
\hline NRAO 512 & $\begin{array}{l}\text { DiFX } \\
\text { Daejeon } \\
S_{\text {Daejeon }} / S_{\text {DiFX }}\end{array}$ & $\begin{array}{c}0.861 \pm 0.163 \\
0.793 \pm 0.188 \\
0.92(1.2)\end{array}$ & $\begin{array}{c}0.908 \pm 0.176 \\
0.839 \pm 0.203 \\
0.92(1.2)\end{array}$ & $\begin{array}{c}0.858 \pm 0.140 \\
0.792 \pm 0.169 \\
0.92(1.2)\end{array}$ & $\begin{array}{c}0.903 \pm 0.153 \\
0.837 \pm 0.183 \\
0.93(1.2)\end{array}$ & $\begin{array}{c}0.837 \pm 0.149 \\
0.768 \pm 0.172 \\
0.92(1.2)\end{array}$ & $\begin{array}{c}0.881 \pm 0.161 \\
0.812 \pm 0.186 \\
0.92(1.2)\end{array}$ \\
\hline
\end{tabular}

Column designation: 1 - source name; 2 - correlator; 3-9 - mean of visibility amplitude for KU-KT, KY-KU, and KT-KY in Jy, or the ratio of the mean values for Daejeon to those for DiFX with the ratio of their standard deviations in parentheses: Columns 3,5,7 for values using data in full bandwidth and Columns 4,6,8 for using data excluding first 6 channels and last 7 channels in each band (hence averaged over 115 channels). 


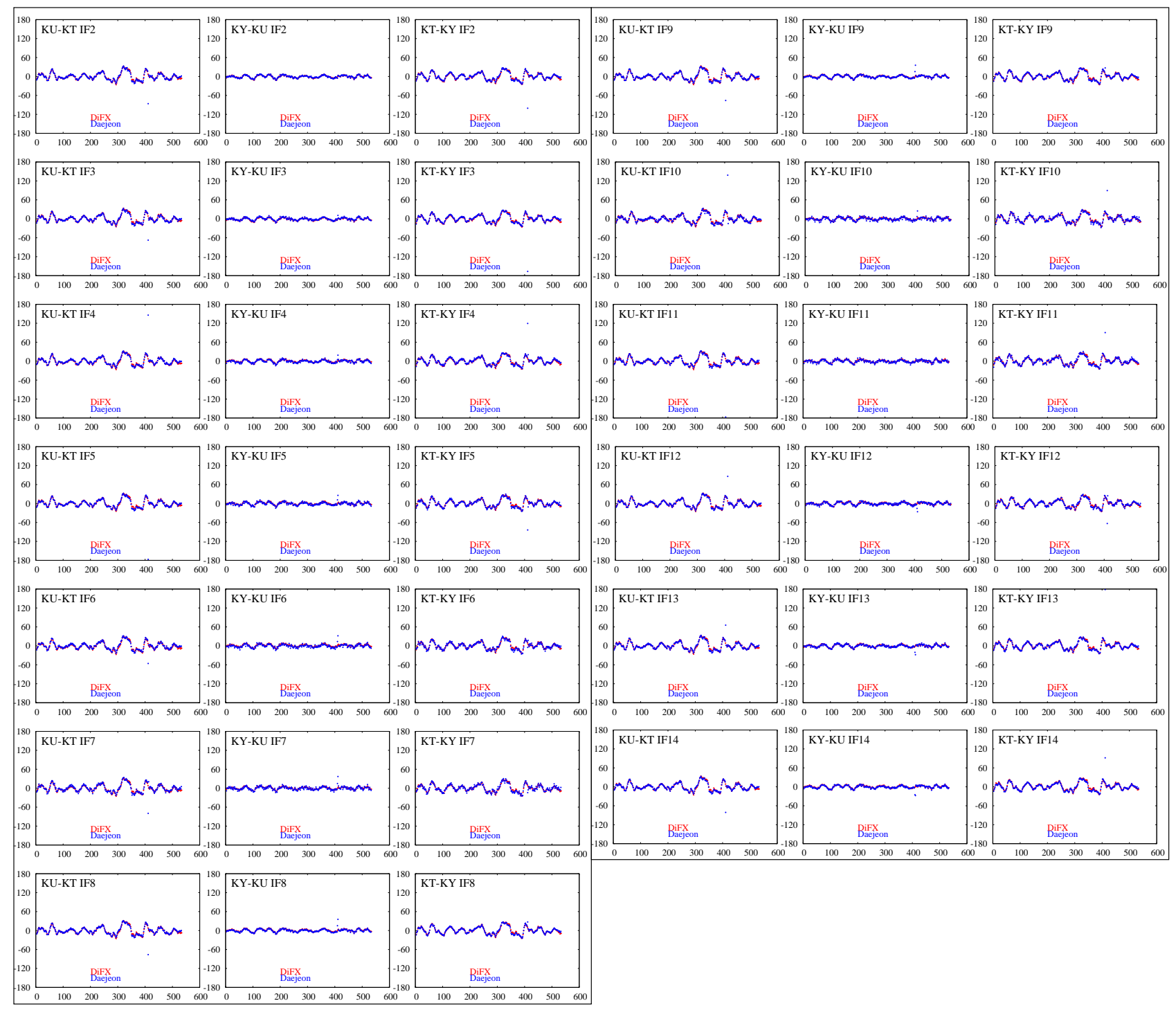

Figure 7. Comparison of visibility phase as a function of time for 3C 454.3 from DiFX (red dots) and the Daejeon correlator (blue dots) outputs for IF 2-14 and three KVN baselines: KY-KT, KY-KU, and KT-KY during 9 min after 2011 January 29 04:20:00 UT. The visibility phase is in degrees and the time is in seconds from the start of the scan.

\subsection{Image Comparison with DiFX}

In addition to the comparison of the visibility amplitudes and phases in time and frequency, we compared the imaging results from the two correlator outputs for all target sources, 3C 454.3, 3C 345, NRAO 512, and $\mathrm{SgrA}^{*}$. As mentioned previously, we used the same procedure of imaging for the comparison: (a) fitting a point source to the visibility, (b) CLEAN and phaseself-calibration, and (c) modelfit with circular Gaussian components. In Figure 9, we compare CLEANed images for the DiFX and Daejeon correlators. We show the contour maps of each source for DiFX in the left panel, and those for Daejeon correlator in the right panel. There are circular Gaussian models on top of the contour maps. The $x$ and $y$ axes are in units of mas. The sources are 3C 454.3, 3C 345, NRAO 512, and $\mathrm{SgrA}^{*}$ from top to bottom. For each contour map, the source name and the observation date are given in the upper left corner of the map. In the lower right corner of the map, we show the peak flux density and the lowest contour level in units of mJy. The shaded ellipse in the lower left corner of the map represents the FWHM of the restoring beam of the image. In all of the images, the contours are drawn at $1,1.4, \ldots, 1.4^{n}$ (logarithmic spacing) of the lowest flux density level. In the right panel, we showed the visibility amplitudes as a function of the $u v$ radius. The $x$ axis of the plot represents the $u v$ radius in units of $10^{6} \lambda$, where $\lambda$ is the observing wavelength, which is the length of the projected baseline used to obtain the visibility data. The $y$ axis of the plot shows the amplitude of each visibility point (i.e., correlated flux density) in units of Jy. The quality of the final images was investigated by estimating the quality of the residual noise, $\xi$, as described in Section 2.3. More detailed parameters of the images 

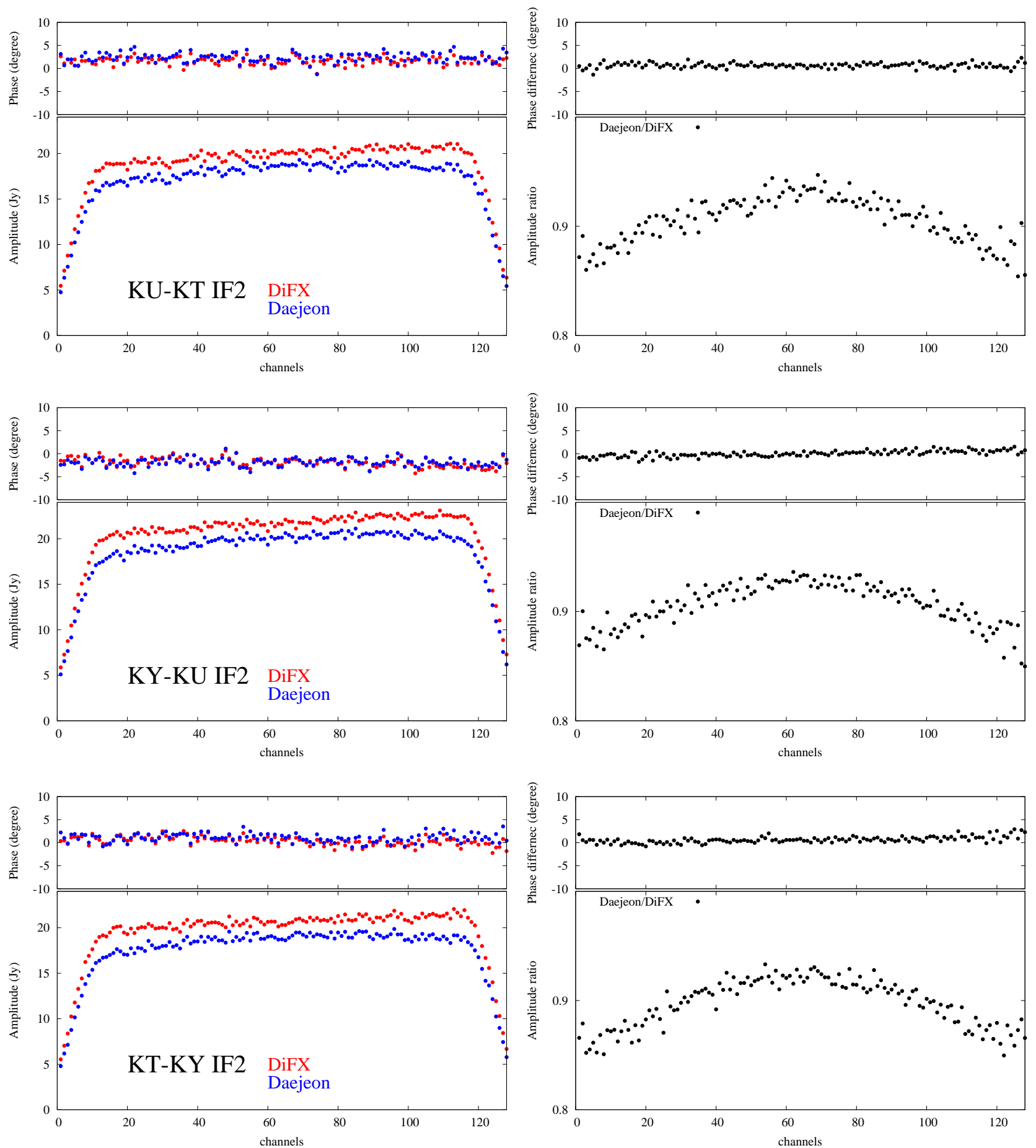

Figure 8. Left: Comparison of the visibility phase (upper panel of each spectrum) and amplitude (lower panel of each spectrum) as a function of frequency for 3C 454.3 from the DiFX (red dots) and the Daejeon correlator (blue dots) outputs for IF 2: 22.034-22.050 GHz band and three KVN baselines: KU-KT (top), KY-KU (middle), and KT-KY (bottom) during 1 min after 2011 January 29 04:20:00 UT. The visibility phase is in degrees spanning a range of $\pm 10^{\circ}$ and the visibility amplitude is in Jy. Right: Phase difference (upper) and amplitude ratio (lower) for two correlators. The phase is shown with the same scale as the left panels while the amplitude ratio spans the range of $0.8-1.0$.

presented in Figure 9 are summarized in Table 2. For each image, Table 2 lists the source name, the parameters of the restoring beam (the size of the major axis, $B_{\text {maj }}$, the minor axis, $B_{\text {min }}$, and the position angle of the beam, $\left.B_{\mathrm{PA}}\right)$, the total flux density, $S_{\mathrm{t}}$, the peak flux density, $S_{\mathrm{p}}$, the off-source rms, $\sigma$, the dynamic range of the image, $D$, and the quality of the residual noise, $\xi$, for each image. The image results show that the total 
flux and peak flux densities for all sources are consistent within $8 \%$ for the two correlator outputs. The difference is similar to that of the visibility amplitude averaged for each baseline as summarized in Table 1. In order to investigate the flux differene in more detail, we made images using data for IF 8 and 9, for which the double-layer pattern does not appear in Figure 4. We compared the total and peak flux density for the images free of the double-layer-pattern, and found that their difference is on average about $5 \%$ for all sources, as summarized in Columns (11) and (12) of Table 2. This may indicate that a part of the total difference of $8 \%$ is caused by other differences between the two correlators that are not related with the double-layer pattern. We also compared the flux density, size, and position of the core and jet components of all sources using circular Gaussian model-fitting. Table 3 lists the parameters of each model-fit component: the total flux, $S_{\mathrm{t}}$, size, $d$, angular distance from the central component, $r$, and position angle, $\theta$ (the location of the jet component with respect to the core component). The modelfit parameters show that the total flux densities of core and jet components of almost all sources are again consistent within $8 \%$ for the two correlator outputs, whereas the sizes of the Gaussian components are different by $\leq 40 \%$. For 3C 454.3 and $3 \mathrm{C} 345$, we were able to modelfit the jet components for the two correlator outputs with very consistent positions to each other.

\section{Discussion}

\subsection{Double-Layer Patterns}

The most prominent, apparent difference between correlation outputs from the Daejeon and DiFX correlators is the double-layer patterns as shown in Figure 4. The double-layer patterns vary in IF (i.e., in frequency) and are present in almost all baselines including KVN (and also VERA stations). When we investigated the visibility amplitude of 3C 454.3 for all baselines of KVN and VERA, we found that the amplitudes for all baselines show the double-layer patterns, except for baselines with larger scatter (e.g., Ishigaki-KVN). For VERA baselines, the double-layer patterns consist of two parallel patterns, and for KVN baselines we see clearly sinusoidal patterns, as discussed above. This is consistent with the results for the KVN baselines of fainter sources.

It is important to investigate possible reasons causing the double-layer pattern and to improve the performance of the Daejeon correlator. A detailed investigation of the reasons behind the double-layer pattern is underway by the KJCC engineering team and will be reported elsewhere. Here we offer a brief discussion of possible ways this pattern can be produced. Possible reasons include the following:

- Fringe rotation error. The plot of the visibility as a function of time shows very periodic patterns, which may indicate some fundamental problem in the correlator: for example, the delay shifter or the phase rotator is not updated as it should. However, as the pattern appears only in the amplitude, it may not be the case. Moreover, same patterns are seen on two separate channels: IF3 $=\mathrm{IF} 14$, $\mathrm{IF} 4=\mathrm{IF} 13, \mathrm{IF} 5=\mathrm{IF} 12, \mathrm{IF} 6=\mathrm{IF} 11, \mathrm{IF} 7=\mathrm{IF} 10 . \quad \mathrm{IF} 8$ and IF9 are both OK, IF2 has no match, and IF1 is not plotted. Since these matching channels have different frequencies, the fringe rotator is not the problem.

- Timing problem between normalization and accumulation. By looking at the plot more closely, we found that some integration intervals may have lost part of the data. The integration time of $1.6384 \mathrm{~s}$ for the Daejeon correlator is so unusual that may modulate with other time in the correlator system. Integer number of seconds of integration time, or shorter intervals, would give better results. It is possible that the IF pattern mentioned above can be compared with the order in which the control computer gets the data from the hardware. We found that this problem does not affect the correlation ouput of DiFX since there is no data loss on the normalization and accumulation in DiFX with the time interval of $2.048 \mathrm{~s}$.

- Problem with the geometry applied in the correlator. The period of the sinusoidal pattern of the double-layer patterns seems to vary in time and in frequency. This could indicate that there is a problem with the geometry applied in the correlator. The correlation output of VLBI observations with a finite bandwidth $\Delta \nu$ is $r \propto \frac{\sin \pi \Delta \nu \tau_{g}}{\pi \Delta \nu \tau_{g}} \cos \left(2 \pi \nu_{0} \tau_{g}-\phi\right)$, where $\tau_{g}$ is the geometric delay and $\nu_{0}$ is the observing frequency (see e.g., Thompson 1999). The amplitude of the correlation output is modulated by a sinc-function envelope as $\frac{\sin \pi \Delta \nu \tau_{g}}{\pi \Delta \nu \tau_{g}}$. Usually the modulation is well modeled by the geometric model used during the correlation. However, if there is an error in geometry, i.e., $\Delta \tau=\tau_{g}-\tau_{m}$, where $\tau_{m}$ is the geometric time delay of the geometric model, then we may expect a periodic pattern in the amplitude of the correlation output equal to $\frac{\sin \pi \Delta \nu \Delta \tau_{g}}{\pi \Delta \nu \Delta \tau_{g}}$. If this is the case, the sinusoidal pattern may correlate with the baseline length. However it is hard to tell wether we see the correlation in the KVN observations, since the baseline length of $\mathrm{KVN}$ is in the range of $305-477 \mathrm{~km}$. Moreover, the geometric problem should affect the phase of the correlation output as $\cos \left(2 \pi \nu_{0} \tau_{g}-\phi\right)$. Since we see no prominent difference in phases between Daejeon and DiFX correlators, we can exclude this possibility.

Although the amplitude of the double-layer patterns is as large as $10-15 \%$ the final effect to the flux density in the CLEANed image is less than $8 \%$ (maybe $3 \%$, see below) because the visibility data are averaged in time and frequency for the final imaging. However this effect should be investigated more carefully with further test observations in various observing modes (e.g., full track 

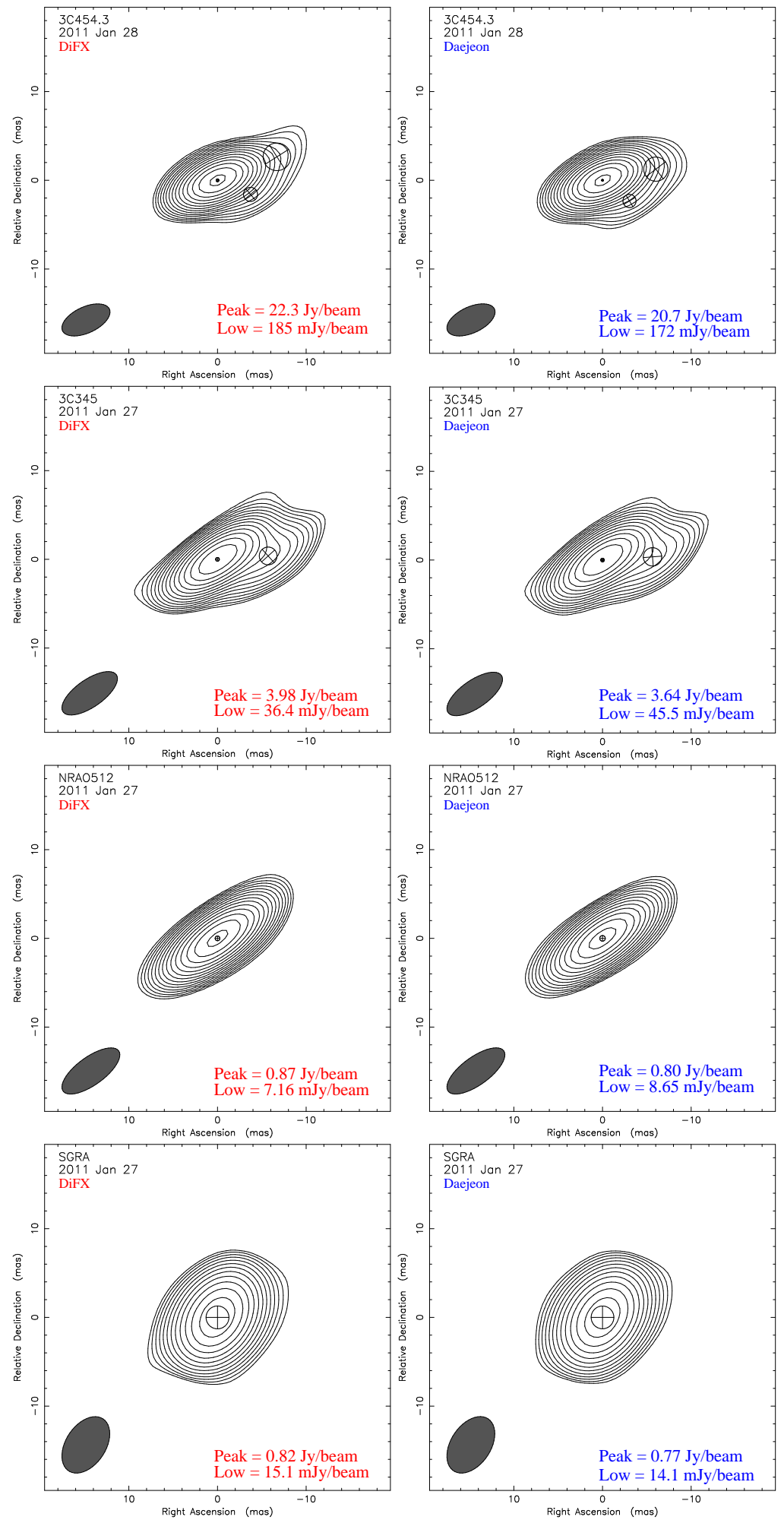
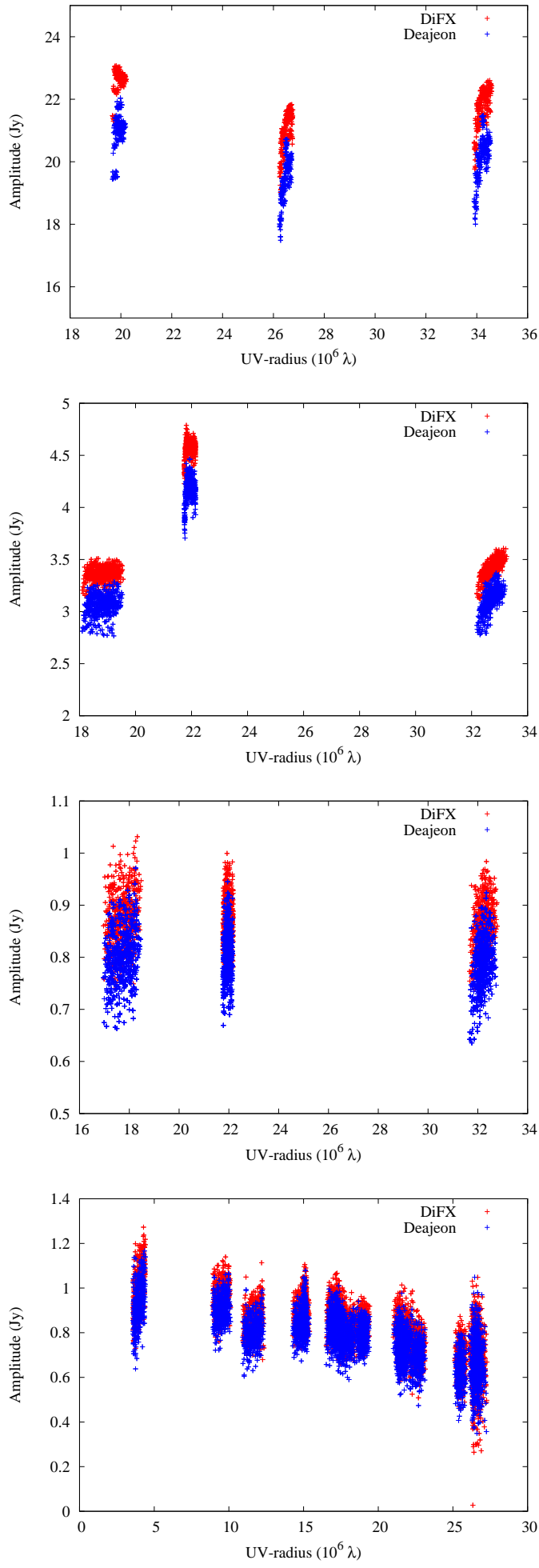

Figure 9. Left: CLEANed images using the DiFX correlator outputs. Circular Gaussian models are on top of the contour maps. The axes of each map are the relative R.A. and decl. offsets from the tracking center in milliarcseconds. The lowest contour level is shown in the lower right corner of each map. The contours have a logarithmic spacing and are drawn at 1 , $1.4, \ldots, 1.4^{n}$ of the lowest contour level. Middle: CLEANed images using the Daejeon correlator outputs in the same format as the left panel. Right: distributions of the visibility amplitude for DiFX (red) and the Daejeon correlator (blue) against the $u v$ radius. The $x$ axis shows the $u v$ distance in $10^{6} \lambda$, and the $y$ axis represents the visibility amplitude (correlated flux density) in Jy, averaged over $30 \mathrm{~s}$. Image parameters of each image are summarized in Table 2. 
Table 2

Comparison of imaging parameters

\begin{tabular}{llcccccccccc}
\hline $\begin{array}{l}\text { Source } \\
(1)\end{array}$ & Correlator & $\begin{array}{c}B_{\operatorname{maj}} \\
(3)\end{array}$ & $\begin{array}{c}B_{\min } \\
(4)\end{array}$ & $\begin{array}{c}B_{\mathrm{PA}} \\
(5)\end{array}$ & $\begin{array}{c}S_{\mathrm{t}} \\
(6)\end{array}$ & $\begin{array}{c}S_{\mathrm{p}} \\
(7)\end{array}$ & $\begin{array}{c}\sigma \\
(8)\end{array}$ & $\begin{array}{c}\mathrm{D} \\
(9)\end{array}$ & $\begin{array}{c}\xi_{r} \\
(10)\end{array}$ & $\begin{array}{c}S_{\mathrm{t}, \text { IF8-9 }} \\
(11)\end{array}$ & $\begin{array}{c}S_{\mathrm{p}, \text { IF8-9 }} \\
(12)\end{array}$ \\
\hline 3C 454.3 & DiFX & 5.829 & 2.937 & -65.1 & 24.10 & 22.27 & 61.53 & 362 & 0.64 & 24.17 & 22.28 \\
& Daejeon & 5.835 & 2.939 & -65.1 & 22.26 & 20.71 & 57.12 & 363 & 0.76 & 23.23 & 21.41 \\
& Daejeon/DiFX & - & - & - & 0.92 & 0.93 & 0.93 & 1.0 & - & 0.96 & 0.96 \\
3C 345 & DiFX & 7.323 & 3.111 & -55.1 & 4.703 & 3.978 & 12.14 & 328 & 0.57 & 4.656 & 4.018 \\
& Daejeon & 7.333 & 3.114 & -55.1 & 4.321 & 3.640 & 15.17 & 240 & 0.56 & 4.390 & 3.790 \\
& Daejeon/DiFX & - & - & - & 0.92 & 0.92 & 1.2 & 0.73 & - & 0.94 & 0.94 \\
NRAO 512 & DiFX & 7.741 & 3.151 & -54.1 & 0.887 & 0.871 & 2.387 & 365 & 0.53 & 0.897 & 0.876 \\
& Daejeon & 7.746 & 3.153 & -54.1 & 0.818 & 0.797 & 2.884 & 276 & 0.53 & 0.855 & 0.829 \\
& Daejeon/DiFX & - & - & - & 0.92 & 0.92 & 1.2 & 0.76 & - & 0.95 & 0.95 \\
Sgr A * & DiFX & 7.033 & 4.493 & -33.5 & 1.011 & 0.822 & 5.023 & 164 & 0.88 & 1.019 & 0.828 \\
& Daejeon & 7.042 & 4.494 & -33.6 & 0.946 & 0.772 & 4.715 & 164 & 0.80 & 0.988 & 0.801 \\
& Daejeon/DiFX & - & - & - & 0.94 & 0.94 & 0.94 & 1.0 & - & 0.97 & 0.97 \\
\hline
\end{tabular}

Column designation: 1 - source name; 2 - correlator; 3 - major axis[mas]; 4 - minor axis $\left[\right.$ mas]; 5 - position angle of the major axis [ ${ }^{\circ}$ ] of the restoring beam; 6 - total flux density [Jy]; 7 - peak flux density [Jy beam ${ }^{-1}$ ], and the ratio of the total and peak flux densities of Daejeon correlator to those of DiFX; 8 - off-source RMS in the image [Jy beam ${ }^{-1}$ ]; 9 - Dynamic range of the image $\left(D=S_{\mathrm{p}} / \sigma\right)$; 10 - quality of the residual noise in the image; 11-12 - total flux density [Jy] and peak flux density [Jy beam ${ }^{-1}$ ] for images using data for IF 8 and 9 .

Table 3

Comparison of model fit parameters

\begin{tabular}{|c|c|c|c|c|c|c|c|c|c|c|}
\hline \multirow[b]{2}{*}{$\begin{array}{l}\text { Source } \\
\text { (1) }\end{array}$} & \multicolumn{2}{|c|}{$S_{\mathrm{t}}$} & \multirow[b]{2}{*}{$\frac{S_{\text {Daejeon }}}{S_{\text {DiFX }}}$} & \multicolumn{2}{|c|}{$d$} & \multirow[b]{2}{*}{$\begin{array}{c}\frac{d_{\text {Daejeon }}}{d_{\text {DiFX }}} \\
(7)\end{array}$} & \multicolumn{2}{|c|}{$r$} & \multicolumn{2}{|c|}{$\theta$} \\
\hline & $\begin{array}{l}\operatorname{DiFX} \\
(2)\end{array}$ & $\begin{array}{c}\text { Daejeon } \\
(3)\end{array}$ & & $\begin{array}{l}\operatorname{DiFX} \\
(5)\end{array}$ & $\begin{array}{c}\text { Daejeon } \\
(6)\end{array}$ & & $\begin{array}{l}\text { DiFX } \\
(8)\end{array}$ & $\begin{array}{c}\text { Daejeon } \\
(9)\end{array}$ & $\begin{array}{c}\text { DiFX } \\
(10)\end{array}$ & $\begin{array}{c}\text { Daejeon } \\
(11)\end{array}$ \\
\hline \multirow[t]{3}{*}{$3 \mathrm{C} 454.3$} & 22.4 & 20.8 & 0.93 & 0.315 & 0.199 & 0.63 & - & - & - & - \\
\hline & 0.872 & 0.835 & 0.96 & 1.59 & 1.48 & 0.93 & 4.05 & 3.83 & -113 & -127 \\
\hline & 0.780 & 0.624 & 0.80 & 3.11 & 2.73 & 0.88 & 7.21 & 6.14 & -68.5 & -78.2 \\
\hline \multirow[t]{2}{*}{$3 \mathrm{C} 345$} & 4.00 & 3.67 & 0.92 & 0.421 & 0.406 & 0.96 & - & - & - & - \\
\hline & 0.702 & 0.648 & 0.92 & 2.00 & 2.07 & 1.0 & 5.72 & 5.66 & -86.2 & -86.4 \\
\hline NRAO 512 & 0.887 & 0.818 & 0.92 & 0.537 & 0.628 & 1.2 & - & - & - & - \\
\hline Sgr A* & 1.01 & 0.946 & 0.94 & 2.58 & 2.56 & 0.99 & - & - & - & - \\
\hline
\end{tabular}

Column designation: 1 - source name; 2-3 - model flux density of the component[Jy]; 4 - ratio of the model flux density of Daejeon correlator to that of the DiFX; 5-6 - size of the component[mas]; 7 - ratio of the model size of the Daejeon correlator to that of DiFX; 8-9 - angular distance from the central component[mas]; 10-11 - position angle $\left.{ }^{\circ}\right]$;

imaging mode). Since the comparison in this paper used the observations with short integration times of 10-20 min (75min for $\mathrm{SgrA}^{*}$ ), we have an uncertainty in imaging results. The image quality values of $\xi_{\mathrm{r}}$ of the final images are in the range of $0.53-0.88$.

\subsection{Fringe Tracking}

We found that part (5\%) of the total difference of $8 \%$ in flux density between the two correlators are caused by other reasons which are not related with the doublelayer pattern. Iguchi et al. (2000) investigated the performance of the hardware correlator developed for the VLBI Space Observatory Programme (VSOP) and found that the loss of visibility amplitude due to the hardware phase tracking is $4 \%$. The loss almost corresponds to the $5 \%$ difference in flux density of images using the double-layer-pattern free data (i.e., data for IF 8 and 9). For the Daejeon correlator, the fringe tracking is done in VCS in the same way as described in Iguchi et al. (2000). So a major part of the $5 \%$ degradation in hardware correlation may come from the difference in the way of fringe phase tracking. The additional $1 \%$ of the difference can be attributed to other reasons (e.g., bit-jump correction).

\section{Conclusions}

We evaluated the performance of a new hardware correlator in Korea, the Daejeon correlator, by conducting KVN VLBI observations at $22 \mathrm{GHz}$. We correlated the acquired data with DiFX and the Daejeon correlator for comparing the visibility data and imaging results. The flux densities and brightness distributions of the target sources for the two correlators are consistent with each other within $<8 \%$. The difference is comparable with the amplitude calibration uncertainties of KVN observations at $22 \mathrm{GHz}$. We also found that the $8 \%$ difference in flux density is caused mainly by (a) the difference in the way of fringe phase tracking between 
the DiFX software correlator and the Daejeon hardware correlator, and (b) an unusual pattern (a double-layer pattern) in the amplitude correlation output from the Daejeon correlator. The visibility amplitude loss by the double-layer pattern is as small as $3 \%$. We conclude that the new hardware correlator produces reasonable correlation outputs for continuum observations which are consistent with the outputs from the software correlator, DiFX.

\section{ACKNOWLEDGMENTS}

We thank Walter Alef, David Graham, and Alan Whitney for their fruitful discussion on the performance of the Daejeon correlator. We would like to thank the anonymous referee for important comments and suggestions which have enormously improved the manuscript. We are grateful to all staff members in KVN who helped to operate the array and to correlate the data. The KVN is a facility operated by the Korea Astronomy and Space Science Institute. The KVN operations are supported by KREONET (Korea Research Environment Open NETwork) which is managed and operated by KISTI (Korea Institute of Science and Technology Information).

\section{REFERENCES}

Bare, C., Clark, B. G., \& Kellermann, K. I. 1967, Interferometer Experiment with Independent Local Oscillators, Science, 157, 189

Carlson, B. R., Dewdney, P. E., Burgess, T. A., et al. 1999, The S2 VLBI Correlator: A Correlator for Space VLBI and Geodetic Signal Processing, PASP, 111,1025

Casse, J. L. 1999, The European VLBI Network MkIV Data Processor, New A Rev., 43, 503

Clark, B. G., Cohnen, M. H., \& Jauncey, D. L. 1967, Angular Size of 3C 273B, ApJ, 149, L151

Clark, B. G. 1973, The NRAO Tape-Recorder Interferometer System, Proc. IEEE, 61, 1242

Deller, A. T., Tingay, S. J., Bailes, M., et al. 2017, DiFX: A Software Correlator for Very Long Baseline Interferometry Using Multiprocessor Computing Environments, PASP, 119, 318

Deller, A. T., Brisken, W. F., Phillips, C. J., et al. 2011, DiFX-2: A More Flexible, Efficient, Robust, and Powerful Software Correlator, PASP, 123, 275

Greisen, E. W. 2009, The FITS Interferometry Data Interchange Convention, AIPS Memo, 114

Horiuchi, S., Kameno, S., Nan, R., et al. 2000, Imaging Capability of the Mitaka VSOP Correlator, Adv. Space Res., 26, 625

Iguchi, S., Kawaguchi, N., Murata, Y., et al. 2000, Development and Performance of the Real-Time VLBI Correlator (RVC), IEICE Trans. Commun., E83-B, 2527

Kondo, T., Koyama, Y., Nakajima, J., et al. 2003, Internet VLBI System Based on the PC-VSSP (IP-
VLBI) Board, in ASP Conf. Ser. 306, New Technologies in VLBI, ed. Y. C. Minh (San Francisco: ASP), 205

Lee, S.-S., Byun, D.-Y., Oh, C. S., et al. 2011, SingleDish Performance of KVN $21 \mathrm{~m}$ Radio Telescopes: Simultaneous Observations at 22 and $43 \mathrm{GHz}$, PASP, 123,1398

Lee, S.-S., Petrov, L., Byun, D.-Y., et al. 2014, Early Science with the Korean VLBI Network: Evaluation of System Performance, AJ, 147, 77

Lobanov, A. P., Krichbaum, T. P., Witzel, A., et al. 2006, Dual-Frequency VSOP Imaging of the Jet in S5 0836+710, PASJ, 58, 253

Moran, J. M., Crowther, P. P., Burke, B. F., et al. 1967, Spectral Line Interferometry with Independent Time Standards at Stations Separated by 845 Kilometers, Science, 157, 676

Napier, P. J., Bargri, D. S., Clark, B. G., et al. 1994, The Very Long Baseline Array, Proc. IEEE, 82, 658

Petrov, L., Lee, S. S., Kim, J., et al. 2012, Early Science with the Korean VLBI Network: The QCAL-1 43 GHz Calibrator Survey, AJ, 144, 150

Rogers, A. E. E., Cappallo, R. J., Hinteregger, H. F., et al. 1983, Very-Long-Baseline Radio Interferometry The Mark III System for Geodesy, Astrometry, and Aperture Synthesis, Science, 219, 51

Ryle, M., \& Hewish, A. 1960, The Synthesis of Large Radio Telescopes, MNRAS, 120, 220

Shepherd, M. C., Pearson, T. J., \& Taylor, G. B. 1994, DIFMAP: an Interactive Program for Synthesis Imaging, BAAS, 26, 987

Shibata, K. M., Kameno, S., Inoue, M., et al. 1998, Mitaka Correlator for the Space VLBI, in ASP Conf. Ser. 144, Radio Emission from Galactic and Extragalactic Compact Sources, ed. J. A. Zensus et al. (San Francisco: ASP), 413

Thompson, A. R. 1999, in Synthesis Imaging in Radio Astronomy II, eds. G. B. Taylor, C. L. Carilli, \& R. A. Perley, ASP Conf. Ser., 180, 11

Whitney, A. R. 1993, The Mark IV VLBI DataAcquisition and Correlation System, in IAU Symp. 156, Developments in Astrometry and Their Impact on Astrophysics and Geodynamics, ed. I. I. Mueller \& B. Kolaczek (Dordrecht: Kluwer), 151

Whitney, A. R. 2002, Mark 5 Disc-Based Gbps VLBI Data System, in Proc. 6th European VLBI Network Symp., New Developments in VLBI Science and Technology, ed. E. Ros et al. (Bonn: Max-PlanckInstitut für Radioastronomie), 41

Wietfeldt, R., Baer, D., Cannon, W. H., et al. 1996, The S2 Very Long Baseline Interferometry Tape Recorder, IEEE Trans. Instrumentation and Measurement, 45, 923

Wilson, W., Roberts, P., \& Davis, E. 1996, in Proc. 4th ATP workshop, ed. E. A. King (Sydney: ATNF/CSIRO), 16 